\title{
On the number of perfect matchings and Hamilton cycles in $\epsilon$-regular non-bipartite graphs
}

\author{
Alan Frieze* \\ Department of Mathematical Sciences \\ Carnegie Mellon University \\ Pittsburgh PA15213, USA \\ alan@random.math. cmu.edu
}

Submitted July 4, 2000, Accepted November 19, 2000

\begin{abstract}
A graph $G=(V, E)$ on $n$ vertices is super $\epsilon$-regular if (i) all vertices have degree in the range $[(d-\epsilon) n,(d+\epsilon) n], d n$ being the average degree, and (ii) for every pair of disjoint sets $S, T \subseteq V,|S|,|T| \geq \epsilon n, e(S, T)$ is in the range $[(d-\epsilon)|S||T|,(d+\epsilon)|S||T|]$. We show that the number of perfect matchings lies in the range $\left[\left((d-2 \epsilon)^{\nu} \frac{n !}{\nu ! 2^{\nu}},(d+2 \epsilon)^{\nu} \frac{n !}{\nu ! 2^{\nu}}\right]\right.$, where $\nu=\frac{n}{2}$, and the number of Hamilton cycles lies in the range $\left[(d-2 \epsilon)^{n} n !,(d+2 \epsilon)^{n} n !\right]$.
\end{abstract}

\section{Introduction}

Let $G=(V, E)$ be a graph with $|V|=n$. Let $0<d<1$ and $\epsilon>0$ be constants (independent of $n$ ) where $\epsilon$ is assumed to be small compared with $d$. We assume that the density of $G$ is $d$ i.e. $|E| /\left(\begin{array}{l}n \\ 2\end{array}\right)=d$. Suppose that the following two conditions hold:

- If $d_{G}$ denotes vertex degree in $G$ then

$$
(d-\epsilon) n \leq d_{G}(v) \leq(d+\epsilon) n \quad \text { for all } v \in V .
$$

${ }^{*}$ Supported in part by NSF Grant CCR9818411

Mathematics Subject Classification (1991); primary 05C50, 05C70, secondary 05C80 
THE ELECTRONiC JOURnAl of COMBinatorics 7 (2000), \#R57

- If for $S, T \subseteq V, S \cap T=\emptyset$ we let $e(S, T)$ denote the number of edges of $G$ with one end in $S$ and the other in $T$ and $d(S, T)=\frac{e(S, T)}{|S||T|}$ then

$$
|d(S, T)-d| \leq \epsilon \text { for all } S, T \subseteq V, S \cap T=\emptyset,|S|,|T| \geq \epsilon n
$$

A graph satisfying (1),(2) said to be super $\epsilon$-regular. We assume that $n=2 \nu$ is even. Let $m(G)$ denote the number of perfect matchings in $G$ and let $h(G)$ denote the number of Hamilton cycles in $G$. In this paper we prove

Theorem 1 If $\epsilon$ is sufficiently small and $n$ is sufficiently large then

(a)

$$
(d-2 \epsilon)^{\nu} \frac{n !}{\nu ! 2^{\nu}} \leq m(G) \leq(d+2 \epsilon)^{\nu} \frac{n !}{\nu ! 2^{\nu}}
$$

(b)

$$
(d-2 \epsilon)^{n} n ! \leq h(G) \leq(d+2 \epsilon)^{n} n !
$$

In both cases the bounds are "close" to the expected number of in the random graph $G_{n, d}$. The results here are strongly related to the result of Alon, Rödl and Ruciński [2]. They considered bipartite graphs $H$ with vertex partition $A, B$ where $|A|=|B|=n$. Assuming (1) and (2) for $S \subseteq A$ and $T \subseteq B$ they proved

Theorem 2 [2]

$$
(d-2 \epsilon)^{n} n ! \leq m(G) \leq(d+2 \epsilon)^{n} n !
$$

Michael Krivelevich has made some interesting observations on Theorem 1: First of all, part (b) of Theorem 1 improves Corollary 2.9 of Thomason [9] which estimates the number of Hamilton cycles in a pseudo-random graph. Secondly, if $G$ is $d n$-regular and the second eigenvalue of the adjacency matrix of $G$ is at most $\eta d n$ for small $\eta$, then $G$ is super $\epsilon(\eta)$-regular (see for example Chung [4] Theorem 5.1) and so our result holds for such graphs.

We note that a similar result can be proven for the number of spanning trees in such a graph: if $t(G)$ denotes the number of spanning trees of $G$ then

$$
(d-2 \epsilon)^{n-1} n^{n-2} \leq t(G) \leq(d+2 \epsilon)^{n-1} n^{n-2} .
$$

This follows from results of Alon [1] and Kostochka [7].

We prove Theorem 1(a) in the next section and Theorem 1(b) in Section 3. For completeness, we also give a proof of (3) in Section 4. 


\section{Perfect Matchings}

Let $A, B,|A|=|B|=\nu$ be a partition of $V$. We re-express (2) in terms of $\nu$ i.e.

$$
|d(S, T)-d| \leq \epsilon \text { for all } S \subseteq A, T \subseteq B,|S|,|T| \geq 2 \epsilon \nu .
$$

Furthermore, if $A, B$ is a random partition and $H=H(A, B)$ is the bipartite sub-graph of $G$ induced by $A, B$ then with high probability $d_{H}(v) \in[(d-\epsilon-o(1)) \nu,(d+\epsilon+o(1)) \nu]$ for all $v \in V$. Thus the conditions of Theorem 2 are satisfied with $\nu$ replacing $n$ and $2 \epsilon$ replacing $\epsilon$. It follows immediately that

$$
m(H) \geq(1-o(1))\left(\begin{array}{l}
n \\
\nu
\end{array}\right) \times \nu !(d-2 \epsilon)^{\nu} \times \frac{1}{2^{\nu}}=(1-o(1)) \frac{n !}{\nu ! 2^{\nu}}(d-2 \epsilon)^{\nu} .
$$

The factor $\frac{1}{2^{\nu}}$ accounts for the fact that each perfect matching occurs in $2^{\nu}$ different graphs $H$, assuming we consider the partition $A, B$ distinct from $B, A$. There is slack in the calculation in [2] and this will absorb the $1-o(1)$ term and so (5) proves the lower bound in Theorem 1.

For the upper bound we follow [2] and use the Minc conjecture [8] proved by Bregman [3]. For a partition $A, B$ and $v \in A$ let $d_{B}(v)$ denote the number of $G$-neighbours of $v$ in $B$. The Minc conjecture then states that

$$
m(H) \leq \prod_{v \in A}\left(d_{B}(v)\right) !^{1 / d_{B}(v)} .
$$

Thus

$$
m(G) \leq \frac{1}{2^{\nu}} \sum_{A, B} \prod_{v \in A}\left(d_{B}(v)\right) !^{1 / d_{B}(v)} .
$$

For a fixed $A$, we let $A_{1}=\left\{v \in A: d_{B}(v)>(d+\epsilon) \nu\right\}$. Property (1) implies that $\left|A_{1}\right| \leq \epsilon n$. Now since $(x !)^{1 / x}$ increases with $x$, we see, after using Stirling's approximation and (1), that

$$
\begin{aligned}
& \prod_{v \in A}\left(d_{B}(v)\right) !^{1 / d_{B}(v)} \leq\left(\frac{d+\epsilon}{e} \nu\right)^{\left|A \backslash A_{1}\right|}\left(\frac{d+\epsilon}{e} n\right)^{\left|A_{1}\right|}\left(1+O\left(\frac{\ln n}{n}\right)\right)^{\nu} \\
& \leq\left(\frac{d+\epsilon}{e} \nu\right)^{\nu} 2^{\epsilon n} n^{O(1)} .
\end{aligned}
$$

Hence

$$
m(G) \leq \frac{1}{2^{\nu}}\left(\begin{array}{l}
n \\
\nu
\end{array}\right)\left(\frac{d+\epsilon}{e} \nu\right)^{\nu} 2^{\epsilon n} n^{O(1)} \leq(d+2 \epsilon)^{\nu} \frac{n !}{\nu ! 2^{\nu}}
$$

completing the proof of part (a) of Theorem 1 . 
THE ELECTRONiC JOURnAl of COMBinatorics 7 (2000), \#R57

\section{Hamilton Cycles}

A Hamilton cycle is the union of two perfect matchings and so $h(G) \leq \frac{1}{2} m(G)^{2}$ and the upper bound in part (b) of Theorem 1 follows from the upper bound in part (a).

The lower bound requires more work. For $1 \leq k \leq\lfloor n / 3\rfloor$, let $\Phi_{k}$ be the set of all 2-factors in $G$ containing exactly $k$ cycles, and let $\Phi=\cup_{k} \Phi_{k}$ be the set of all 2-factors. Let $f_{k}=\left|\Phi_{k}\right|$ so that $f_{1}=h(G)$. If $M$ is a perfect matching of $G$, let $a_{M}$ denote the number of perfect matchings of $G$ that are disjoint from $M$. Since deleting $M$ only disturbs $\epsilon$-regularity marginally, we see by part (a) that $a_{M} \geq(d-2 \epsilon)^{\nu} \frac{n !}{\nu ! 2^{\nu}}$. Thus

$$
A_{G}=\sum_{M \in G} a_{M} \geq\left((d-2 \epsilon)^{\nu} \frac{n !}{\nu ! 2^{\nu}}\right)^{2} \geq(d-2 \epsilon)^{n} n ! \times \frac{1}{3 n^{1 / 2}} .
$$

On the other hand, we have

$$
A_{G} \leq \sum_{k=1}^{\lfloor n / 3\rfloor} 2^{k} f_{k}
$$

We will show by a relatively crude argument that where $k_{1}=\left\lceil\frac{4}{(d-2 \epsilon)(d-\epsilon)}\right\rceil$

$$
\frac{f_{k+1}}{f_{k}} \leq n^{3} \quad 1 \leq k \leq k_{1}
$$

We then use an idea from Dyer, Frieze and Jerrum [5]. In this paper they show that if an $n$ vertex graph $G$ has minimum degree $\delta(G) \geq\left(\frac{1}{2}+\alpha\right) n$ for a positive constant $\alpha$, then a polynomial fraction of the 2-factors of $G$ are Hamilton cycles. We extend their argument to $\epsilon$-regular graphs.

Let $\beta=\frac{200}{(d-2 \epsilon)(d-\epsilon)^{2}}$. Let $k_{0}=\lfloor\beta \ln n\rfloor$, and for $1 \leq k \leq n$, define $\gamma(k)=$ $n^{\beta} k !(\beta \ln n)^{-k}$, and

$$
\phi(k)= \begin{cases}\gamma(k), & \text { if } k \leq k_{0} \\ \gamma\left(k_{0}\right), & \text { otherwise }\end{cases}
$$

Lemma 1 Let $\phi$ be the function defined above. Then

1. $\phi$ is non-increasing and satisfies

$$
\min \{\phi(k-1), \phi(k-2)\}=\phi(k-1) \geq(\beta \ln n) k^{-1} \phi(k) ;
$$

2. $\phi(k) \geq 1$, for all $k$.

Proof Observe that $\gamma$ is unimodal, and that $k_{0}$ is the value of $k$ minimizing $\gamma(k)$; it follows that $\phi$ is non-increasing. When $k \leq k_{0}$, we have $\phi(k-1)=\gamma(k-1)=$ $(\beta \ln n) k^{-1} \gamma(k)=(\beta \ln n) k^{-1} \phi(k)$; otherwise, $\phi(k-1)=\gamma\left(k_{0}\right)=\phi(k) \geq(\beta \ln n) k^{-1} \phi(k)$. In either case, the inequality in part 1 of the lemma holds. 
Part 2 of the lemma follows from the chain of inequalities

$$
\frac{1}{\phi(k)} \leq \frac{1}{\gamma\left(k_{0}\right)} \leq \frac{(\beta \ln n)^{k_{0}}}{n^{\beta} k_{0} !} \leq n^{-\beta} \sum_{k=0}^{\infty} \frac{(\beta \ln n)^{k}}{k !}=n^{-\beta} \exp (\beta \ln n)=1 .
$$

Define

$$
\Psi=\left\{\left(F, F^{\prime}\right): F \in \Phi_{k}, F^{\prime} \in \Phi_{k^{\prime}}, k^{\prime}<k \text {, and } F \oplus F^{\prime} \text { is a 6-cycle }\right\},
$$

where $\oplus$ denotes symmetric difference. Observe that $\Gamma=(\Phi, \Psi)$ is an acyclic directed graph; let us agree to call its component parts nodes and arcs to avoid confusion with the vertices and edges of $G$. Observe also that if $\left(F, F^{\prime}\right) \in \Psi$ is an arc, then $F^{\prime}$ can be obtained from $F$ by deleting three edges and adding three others, and that this operation can decrease the number of cycles by at most two. Thus every arc $\left(F, F^{\prime}\right) \in \Psi$ is directed from a node $F$ in some $\Phi_{k}$ to a node $F^{\prime}$ in $\Phi_{k-1}$ or $\Phi_{k-2}$.

Our proof strategy is to define a positive weight function $w$ on the arc set $\Psi$ such that the total weight of arcs leaving each node (2-factor) $F \in \Phi_{\geq k_{1}}$ is significantly greater than the total weight of arcs entering $F$. We will show below that

$$
\begin{array}{ll}
\sum_{F^{+}:\left(F, F^{+}\right) \in \Psi} w\left(F, F^{+}\right) \geq 100 \phi(k) n^{2} \ln n & F \in \Phi_{k}, k \geq k_{1}, \\
\sum_{F^{-}:\left(F^{-}, F\right) \in \Psi} w\left(F^{-}, F\right) \leq 9 \phi(k) n^{2} H_{n} & F \in \Phi_{k}, k \geq 1,
\end{array}
$$

where $H_{n}=\sum_{i=1}^{n} i^{-1} \leq \ln n+1$ is the $n$th harmonic number.

Now let

$$
W_{k, l}=\sum_{\substack{F \in \Phi_{k}, F^{\prime} \in \Phi_{l} \\\left(F, F^{\prime}\right) \in \Psi}} w\left(F, F^{\prime}\right)
$$

Then (10) and (11) imply that for $k \geq k_{1}$,

$$
\begin{aligned}
& W_{k+2, k}+W_{k+1, k} \leq 9 f_{k} \phi(k) n^{2} H_{n} \\
& W_{k, k-1}+W_{k, k-2} \geq 100 f_{k} \phi(k) n^{2} \ln n .
\end{aligned}
$$

Now (13) implies that either

(i) $W_{k, k-1} \geq 50 f_{k} \phi(k) n^{2} \ln n$ so that from (12)(k-1) we have

$$
\frac{f_{k-1}}{f_{k}} \geq 5 \frac{\phi(k)}{\phi(k-1)}
$$

or

(ii) $W_{k, k-2} \geq 50 f_{k} \phi(k) n^{2} \ln n$ so that from (12)(k-2) we have

$$
\frac{f_{k-2}}{f_{k}} \geq 5 \frac{\phi(k)}{\phi(k-2)}
$$


It follows that if $k \geq k_{0}+2$ then

$$
f_{k} \leq 5^{-\left(k-k_{0}\right) / 2} \max \left\{f_{k_{0}+1}, f_{k_{0}}\right\} .
$$

Then from (8) we see that

$$
A_{G} \leq \frac{\sqrt{5}}{\sqrt{5}-2} \sum_{k=1}^{k_{0}+1} 2^{k} f_{k} \leq \frac{\sqrt{5}}{\sqrt{5}-2} 2^{k_{0}+1} \sum_{k=1}^{k_{0}+1} f_{k}
$$

Furthermore, since $F \in \Phi_{k}, k>k_{1}$ implies that

$$
\sum_{F^{+}:\left(F, F^{+}\right) \in \Psi} w\left(F, F^{+}\right)-\sum_{F^{-}:\left(F^{-}, F\right) \in \Psi} w\left(F^{-}, F\right) \geq 1
$$

the total weight of arcs entering $\Phi_{k_{1}}$ is an upper bound on the number of 2-factors in $G$ with more than $k_{1}$ cycles and the maximum total weight of arcs entering a single node in $\Phi_{k_{1}}$ is an upper bound on the ratio $\rho=\frac{f_{k_{1}+1}+f_{k_{1}+2}+\cdots+f_{\lfloor n / 3\rfloor}}{f_{k_{1}}}$. Thus

$$
\rho \leq 9 \phi(1) n^{2} H_{n}=O\left(n^{2+\beta}\right) .
$$

Combined with (14) and (9) we see that

$$
A_{G} \leq n^{O(1)} f_{1}
$$

and the lower bound in Theorem 1(b) follows from (7), modulo taking advantage of slack to absorb the $n^{O(1)}$ term.

\subsection{Proofs of (10) and (11)}

The weight function $w: \Psi \rightarrow \mathbf{R}^{+}$we employ is defined as follows. For any $\operatorname{arc}\left(F^{\prime}, F\right)$ with $F \in \Phi_{k}$ : if the 2-factor $F$ is obtained from $F^{\prime}$ by coalescing two cycles of lengths $l_{1}$ and $l_{2}$ into a single cycle of length $l_{1}+l_{2}$, then $w\left(F^{\prime}, F\right)=\left(l_{1}^{-1}+l_{2}^{-1}\right) \phi(k)$; if $F$ results from coalescing three cycles of length $l_{1}, l_{2}$ and $l_{3}$ into a single one of length $l_{1}+l_{2}+l_{3}$, then $w\left(F^{\prime}, F\right)=\left(l_{1}^{-1}+l_{2}^{-1}+l_{3}^{-1}\right) \phi(k)$.

Let $F \in \Phi_{k}$ be a 2-factor with $k>1$ cycles $C_{1}, C_{2}, \ldots, C_{k}$, of lengths $n_{1}, n_{2}, \ldots, n_{k}$. We proceed to bound from below the total weight of arcs leaving $F$. For this purpose imagine that the cycles $C_{1}, C_{2}, \ldots, C_{k}$ are oriented in some way, so that we can speak of each oriented edge $\left(u, u^{\prime}\right)$ in some cycle $C_{i}$ as being "forward" or "backward". For each vertex $a$ we can then let $(a, \pi(a))$ be the unique forward edge containing $a$. Since we are interested in obtaining a lower bound, it is enough to consider only $\operatorname{arcs}\left(F, F^{+}\right)$ from $F$ of a certain kind: namely, those for which the 6-cycle $C=F \oplus F^{+}$is of the form $C=\left(x, x^{\prime}, y, y^{\prime}, z, z^{\prime}\right)$, where $\left(x, x^{\prime}\right) \in F$ is a forward cycle edge, $\left(y, y^{\prime}\right) \in F$ is a forward edge in a cycle distinct from the first, and $\left(z, z^{\prime}\right) \in F$ is a backward cycle edge. The edge $\left(z, z^{\prime}\right)$ may be in the same cycle as either $\left(x, x^{\prime}\right)$ or $\left(y, y^{\prime}\right)$, or in a third cycle. Observe 
that $\left(x^{\prime}, y\right),\left(y^{\prime}, z\right)$ and $\left(z^{\prime}, x\right)$ must necessarily be edges of $F^{+}$. It is routine to check that any cycle $C=\left(x, x^{\prime}, y, y^{\prime}, z, z^{\prime}\right)$ satisfying the above constraints does correspond to a valid arc from $F$. The fact that $\left(z, z^{\prime}\right)$ is oriented in the opposite sense to $\left(x, x^{\prime}\right)$ and $\left(y, y^{\prime}\right)$ plays a crucial role in ensuring that the number of cycles decreases in the passage to $F^{+}$when only two cycles are involved.

First, we estimate the number of cycles $C$ for which a fixed $\left(x, x^{\prime}\right)$ is contained in a particular cycle $C_{i}$ of $F$. We say that $C$ is rooted at $C_{i}$. Let $Z^{\prime}$ be the neighbour set of $x$ in $G$ and $Z=\pi\left(Z^{\prime}\right)$. Similarly, let $Y^{\prime}$ be the set of neighbours of $x^{\prime}$ which do not belong to $C_{i}$ and let $Y=\pi\left(Y^{\prime}\right)$. If $\left|Y^{\prime}\right| \geq \epsilon n$ then by $\epsilon$-regularity there are at least $(d-2 \epsilon) n$ vertices $z \in Z$ which have at least $(d-\epsilon)\left|Y^{\prime}\right| \geq(d-\epsilon)\left((d-\epsilon) n-n_{i}\right)$ neighbours $y^{\prime}$ in $Y^{\prime}$. Let $\delta_{i}=1_{n_{i} \leq(d-2 \epsilon) n}$. We see that $\delta_{i}=1$ implies $\left(x, x^{\prime}\right)$ is contained in at least $(d-2 \epsilon)(d-\epsilon)\left((d-\epsilon) n-n_{i}\right) n$ cycles. Note also that $\sum_{i=1}^{k} \delta_{i} \geq k-\frac{1}{d-2 \epsilon}$.

We can now bound the total weight of arcs leaving $F$. Each $\operatorname{arc}\left(F, F^{+}\right)$defined by a cycle $C$ rooted at $C_{i}$ has weight at least $n_{i}^{-1} \min \{\phi(k-1), \phi(k-2)\}$, which, by Lemma 1 , is bounded below by $(\beta \ln n)\left(k n_{i}\right)^{-1} \phi(k)$. Thus the total weight of arcs leaving $F$ is bounded as follows:

$$
\begin{aligned}
\sum_{F^{+}:\left(F, F^{+}\right) \in \Psi} w\left(F, F^{+}\right) & \geq \sum_{i=1}^{k}(d-2 \epsilon)(d-\epsilon)\left((d-\epsilon) n-n_{i}\right) n \delta_{i} n_{i} \frac{(\beta \ln n) \phi(k)}{k n_{i}} \\
& \geq \beta(d-2 \epsilon)(d-\epsilon) \phi(k)\left(d-\epsilon-\frac{1}{k(d-2 \epsilon)}-\frac{1}{k}\right) n^{2} \ln n(17 \\
& \geq \beta(d-2 \epsilon)(d-\epsilon) \phi(k) \frac{d-\epsilon}{2} n^{2} \ln n \\
& \geq 100 \phi(k) n^{2} \ln n
\end{aligned}
$$

where we have used the fact that $k \geq k_{1}$. Note that the presence of a unique backward edge, namely $\left(z, z^{\prime}\right)$, ensures that each cycle $C$ has a distinguishable root, and hence that the arcs $\left(F, F^{+}\right)$were not overcounted in summation (16). This completes the proof of (10).

We now turn to the corresponding upper bound on the total weight of $\operatorname{arcs}\left(F^{-}, F\right) \in$ $\Psi$ entering $F$. It is straightforward to verify that the cycle $C=\left(x, x^{\prime}, y, y^{\prime}, z, z^{\prime}\right)=$ $F^{-} \oplus F$ must contain three edges - $\left(x, x^{\prime}\right),\left(y, y^{\prime}\right)$ and $\left(z, z^{\prime}\right)$ - from a single cycle $C_{i}$ of $F$, the remaining edges coming from $F^{-}$. The labeling of vertices in $C$ can be made canonical in the following way: assume an ordering on vertices in $V$, and assign label $x$ to the smallest vertex. The condition $\left(x, x^{\prime}\right) \in F$ uniquely identifies vertex $x^{\prime}$, and the labeling of the other vertices in the cycle $C$ follows.

Removing the three edges $\left(x, x^{\prime}\right),\left(y, y^{\prime}\right)$ and $\left(z, z^{\prime}\right)$ from $C_{i}$ leaves a triple of simple paths of lengths (say) $a-1, b-1$ and $c-1$ : these lengths correspond (respectively) to the segment containing $x$, the segment containing $x^{\prime}$, and the remaining segment. Going round the cycle $C_{i}$, starting at $x^{\prime}$ and ending at $x$, the vertices $x, x^{\prime}, y, y^{\prime}, z, z^{\prime}$ may appear in one of eight possible sequences:

$$
x^{\prime}, y^{\prime}, y, z^{\prime}, z, x ;
$$




$$
\begin{aligned}
& x^{\prime}, z, z^{\prime}, y, y^{\prime}, x ; \\
& x^{\prime}, z, z^{\prime}, y^{\prime}, y, x ; \\
& x^{\prime}, z^{\prime}, z, y, y^{\prime}, x ; \\
& x^{\prime}, y^{\prime}, y, z, z^{\prime}, x ; \\
& x^{\prime}, y, y^{\prime}, z^{\prime}, z, x ; \\
& x^{\prime}, z^{\prime}, z, y^{\prime}, y, x ; \\
& x^{\prime}, y, y^{\prime}, z, z^{\prime}, x .
\end{aligned}
$$

For a given triple of lengths $(a, b, c)$, each of the above sequences corresponds to at most $n_{i}$ possible choices for the edges $\left(x, x^{\prime}\right),\left(y, y^{\prime}\right)$ and $\left(z, z^{\prime}\right)$, yielding a maximum of $8 n_{i}$ in total. To see this, observe that the edge $\left(x, x^{\prime}\right)$ may be chosen in $n_{i}$ ways (minimality of $x$ fixes the orientation of the edge), and that the choice of $\left(x, x^{\prime}\right)$ combined with the information provided by the sequence completely determines the triple of edges.

The eight sequences divide into five possible cases, as the first four sequences lead to equivalent outcomes (covered by case 1 below). Taken in order, the five cases are:

1. For at most $4 n_{i}$ of the choices for the edges $\left(x, x^{\prime}\right),\left(y, y^{\prime}\right)$ and $\left(z, z^{\prime}\right), C_{i} \oplus C$ is a single cycle;

2. for at most $n_{i}$ choices, $C_{i} \oplus C$ is a pair of cycles of lengths $b$ and $a+c$;

3. for at most $n_{i}$ choices, $C_{i} \oplus C$ is a pair of cycles of lengths $a$ and $b+c$;

4. for at most $n_{i}$ choices, $C_{i} \oplus C$ is a pair of cycles of lengths $c$ and $a+b$;

5. for at most $n_{i}$ choices, $C_{i} \oplus C$ is a triple of cycles of lengths $a, b$ and $c$.

The first case does not yield an arc $\left(F^{-}, F\right)$, since the number of cycles does not decrease when passing from $F^{-}=F \oplus C$ to $F$, but the other four cases do have to be reckoned with.

The total weight of arcs entering $F$ can be bounded above as follows:

$$
\begin{aligned}
\sum_{F^{-}:\left(F^{-}, F\right) \in \Psi} w\left(F^{-}, F\right) \leq & \sum_{i=1}^{k} n_{i} \phi(k) \sum_{\substack{a, b, c \geq 1 \\
a+b+c=n_{i}}}\left[\left(\frac{1}{a}+\frac{1}{b}+\frac{1}{c}\right)+\right. \\
& \left.\left(\frac{1}{a}+\frac{1}{b+c}\right)+\left(\frac{1}{b}+\frac{1}{a+c}\right)+\left(\frac{1}{c}+\frac{1}{a+b}\right)\right] \\
& =\sum_{i=1}^{k} n_{i} \phi(k) \sum_{\substack{a, b, c \geq 1 \\
a+b+c=n_{i}}}\left[\frac{6}{a}+\frac{3}{b+c}\right] \\
\leq & \sum_{i=1}^{k} n_{i} \phi(k) n \sum_{a=1}^{n_{i}-1}\left[\frac{6}{a}+\frac{3}{n_{i}-a}\right] \\
\leq & 9 \phi(k) n^{2} H_{n} .
\end{aligned}
$$

This completes the proof of (11). 
THE ElECtronic JoURnal of COMBInAtorics 7 (2000), \#R57

\section{$3.2 \quad$ Proof of $(9)$}

We show that if $F \in \Phi_{k}$ and $2 \leq k \leq k_{1}$ then there is at least one arc $\left(F, F^{\prime}\right) \in \Psi$. Since each $F^{\prime}$ is the terminus of at most $n^{3}$ arcs, (9) follows immediately.

Let $C_{1}$ be the largest cycle of $F$. Then $\left|C_{1}\right| \geq n / k_{1} \geq \frac{d^{2}}{5} n$.

Case 1: $\left|C_{1}\right| \leq n-3 \epsilon n$.

$\epsilon$-regularity implies that there are at most $\epsilon n$ vertices $X$ which have fewer than $(d-\epsilon)\left|C_{1}\right|$ neighbours in $C_{1}$. As there are at least $3 \epsilon n$ vertices not in $C_{1}$, there are vertices $x_{1}, x_{2} \notin X$ which are neighbours on a cycle $C_{2} \neq C_{1}$. Let $A_{i}, i=1,2$ be the neighbour sets of $x_{i}$ on $C_{1}$ and let $B_{i}=\pi\left(A_{i}\right)$ for $i=1,2$. By assumption, $\left|B_{i}\right| \geq \frac{1}{5} d^{2}(d-\epsilon) n$ for $i=1,2$ and so we can choose $B_{i}^{\prime} \subseteq B_{i}, i=1,2$ such that $B_{1}^{\prime} \cap B_{2}^{\prime}=\emptyset$ and $\left|B_{1}^{\prime}\right|=\left|B_{2}^{\prime}\right| \geq \frac{1}{6} d(d-\epsilon) n$. $\epsilon$-regularity implies that there is at least one edge joining $B_{1}^{\prime}, B_{2}^{\prime}$. Suppose this is the edge $\left(b_{1}, b_{2}\right)$. Then $x_{1}, \pi^{-1}\left(b_{1}\right), b_{1}, b_{2}, \pi^{-1}\left(b_{2}\right), x_{2}, x_{1}$ defines the requisite 6 -cycle.

Case 2: $\left|C_{1}\right|>n-3 \epsilon n$.

Just take any two vertices which are neighbours on a cycle other than $C_{1}$. Each has at least $(d-4 \epsilon) n$ neighbours in $C_{1}$ and we can argue the existence of a 6 -cycle as in the previous case.

Remark: The proof shows that the number of Hamilton cycles is within a polynomial factor of the number of two factors of $G$. Therefore one can generate a (near) random Hamilton cycle of $G$ by generating (near) random 2-factors of $G$ until a Hamilton cycle is produced. By doing this sufficiently many times we expect to obtain a good approximation to the ratio of Hamilton cycles to 2-factors. Since the number of 2-factors of $G$ can be efficiently approximated to within arbitrary accuracy (Jerrum, Sinclair and Vigoda [6]) we see that we can efficiently estimate the number of Hamilton cycles of $G$ to within arbitrary accuracy. Formally, there is a Fully Polynomial Time Randomised Approximation Scheme for estimating the number of Hamilton cycles in an super $\epsilon$-regular graph. It is of course assume that $d>0$ is constant and $\epsilon<d$. This same argument is used in [5].

\section{Spanning Trees}

For the lower bound let $\Omega=\{f: V \rightarrow V:(v, f(v)) \in E$, for all $v \in V\}$ be the set of functions defined by each $v \in V$ choosing a neighbour $f(v)$. Clearly

$$
|\Omega|=\prod_{v \in V} d_{G}(v) \geq(d-\epsilon)^{n} n^{n}
$$

Each $f \in \Omega$ defines a digraph $D_{f}=\left(V, A_{f}\right), A_{f}=\{(v, f(v): v \in V\}$. A weak component of $D_{f}$ consists of a cycle $C$ with a rooted forest whose roots are in $C$. Suppose that $D_{f}$ has $k_{f}$ weak components. We obtain a spanning tree of $G$ by (i) deleting the lexicographically first edge of each cycle of $D_{f}$ and then (ignoring orientation) extending 
the $k_{f}$ components to a spanning tree. We claim that if $\alpha=4 / \sqrt{d-\epsilon}$ and

$$
\Omega_{1}=\left\{f \in \Omega: k_{f} \leq \alpha \sqrt{n}\right\}
$$

then

$$
\left|\Omega_{1}\right| \geq|\Omega| / 2
$$

Assume that (20) holds. Each spanning tree is obtained by deleting $k_{f}$ edges of a $D_{f}$ and then adding $k_{f}-1$ edges. It follows that each spanning tree can be obtained in at most $\left(\begin{array}{c}N \\ \alpha \sqrt{n}\end{array}\right)^{2}, N=\left(\begin{array}{c}n \\ 2\end{array}\right)$ ways from a member of $\Omega_{1}$. Thus

$$
t(G) \geq \frac{1}{2} n^{-4 \alpha \sqrt{n}}(d-\epsilon)^{n} n^{n}
$$

and the lower bound in (3) follows.

Proof of (20)

Let $f$ be chosen randomly from $\Omega$ and write

$$
k_{f}=\sum_{v \in V} \frac{1}{\left|K_{v}\right|}
$$

where $K_{v}$ is the weak component containing $v$.

We will argue that

$$
\operatorname{Pr}\left(\left|K_{v}\right| \leq k\right) \leq \frac{k^{2}}{(d-\epsilon) n} \quad k \geq 1
$$

Given (21) we have

$$
\begin{aligned}
\mathbf{E}\left(\left|K_{v}\right|^{-1}\right) & \leq \sum_{k=1}^{\sqrt{(d-\epsilon) n}} \frac{1}{k}\left(\operatorname{Pr}\left(\left|K_{v}\right| \leq k\right)-\operatorname{Pr}\left(\left|K_{v}\right| \leq k-1\right)\right)+\frac{1}{\sqrt{(d-\epsilon) n}} \\
& \leq \sum_{k=1}^{\sqrt{(d-\epsilon) n}} \frac{\operatorname{Pr}\left(\left|K_{v}\right| \leq k\right)}{k(k+1)}+\frac{1}{\sqrt{(d-\epsilon) n}} \\
& \leq \sum_{k=1}^{\sqrt{(d-\epsilon) n}} \frac{1}{(d-\epsilon) n}+\frac{1}{\sqrt{(d-\epsilon) n}} \\
& =\frac{2}{\sqrt{(d-\epsilon) n}}
\end{aligned}
$$

Thus

$$
\mathbf{E}\left(k_{f}\right) \leq \frac{2 \sqrt{n}}{\sqrt{d-\epsilon}}
$$


and (20) follows from the Markov inequality.

To verify (21), start with $v$ and follow $v, f(v), f^{2}(v), \ldots$, until there is a repetition in the sequence. The probability of a repetition at the $i$ th step is at most $\frac{i}{(d-\epsilon) n}$, since there are always at least $(d-\epsilon) n$ random choices for $f^{i}(v)$. If there are no repetitions by step $k$ then $\left|K_{v}\right|>k$. Thus

$$
\operatorname{Pr}\left(\left|K_{v}\right| \leq k\right) \leq \sum_{i=1}^{k} \frac{i}{(d-\epsilon) n}
$$

and (21) follows.

For the upper bound in (3) let $\Omega^{*}=\{f: V \rightarrow V:(v, f(v)) \in E$ or $f(v)=v$ for all $v \in$ $V\}$. Then

$$
t(G) \leq\left|\Omega^{*}\right| \leq((d+\epsilon) n+1)^{n} \leq(d+2 \epsilon)^{n-1} n^{n-2} .
$$

To see this consider the following injective map from the spanning trees of $G$ into $\Omega^{*}$ : orient each edge of tree $T$ towards vertex 1 and then put $f(1)=1$.

Acknowledgement We thank Michael Krivelevich for his earlier comments and for pointing out the simple proof of the upper bound in (3).

\section{References}

[1] N. Alon, The number of spanning trees in regular graphs, Random Structures and Algorithms 1 (1990) 175-182.

[2] N. Alon, V. Rödl and A. Ruciński, Perfect matchings in e-regular graphs, The Electronic Journal of Combinatorics 5 (1998) \#R13.

[3] L. M. Bregman, Some properties of non-negative matrices and their permanents, Soviet Mathematics Doklady 14 (1973) 945-949.

[4] F. R. K. Chung, Spectral Graph Theory, CBMS monograph no. 92, American Mathematical Society 1997.

[5] M. E. Dyer, A. M. Frieze and M. R. Jerrum, Approximately counting Hamilton cycles in dense graphs, SIAM Journal on Computing 27 (1998) 1262-1272.

[6] M. R. Jerrum, A. Sinclair and E. Vigoda, A polynomial-time approximation algorithm for the permanent of a matrix with non-negative entries, to appear.

[7] A. V. Kostochka, The number of spanning trees in graphs with given degree sequences, Random Structures and Algorithms 6 (1995) 269-274.

[8] H. Minc, Non-negative matrices, Wiley, 1988.

[9] A. Thomason, Pseudo-random graphs, Proceedings of Random Graphs 85, Annals of Discrete Mathematics 33, M. Karonski, Z. Palka, eds., North Holland (1985) 307-331. 